\title{
PIV INVESTIGATION OF COUETTE-TAYLOR FLOWS WITH AXIAL FLOWS
}

\section{Emna BERRICH}

Emna.berrich@mines-nantes.fr

LUNAM Université, GEPEA, CNRS, UMR6144, École des Mines de Nantes, DSEE

4 rue Alfred Kastler - BP20722

44307 Nantes Cedex 03 - France

\author{
* Fethi ALOUI \\ * Corresponding author: \\ Fethi.aloui@univ-valenciennes.fr \\ LAMIH UMR CNRS 8201, University \\ of Valenciennes (UVHC) \\ Department of Mechanics, Campus \\ Le Mont Houy \\ 59313 Valenciennes Cedex 9 - \\ France
}

\author{
Jack LEGRAND \\ Jack.Legrand@univ-nantes.fr \\ LUNAM Université, Université de \\ Nantes, CNRS, GEPEA, UMR6144, \\ CRTT, 37 Boulevard de l'Université \\ BP 406, 44602 Saint-Nazaire Cedex - \\ France
}

\section{ABSTRACT}

The Couette Taylor flows CTF strongly depend on geometrical characteristics of CT systems radio and aspect ratios. The superposition of axial flow may accentuate this dependence. Previous studies carried with relatively small radial and/or aspect ratios [1-3] or relatively low Taylor numbers (or rotational Reynolds number $\mathrm{Re}_{\Omega}$ ) and/or low axial flux rates (exp. [4]: $\operatorname{Re}_{\Omega}<50$ and $\operatorname{Re}_{\mathrm{ax}}<400$; [1] : $\operatorname{Re}_{\mathrm{ax}}<4$ ), or limited to analytical approaches or numerical simulations adopting simplified hypothesis and assumptions. In order to complete information obtained for vortices characterization for relatively "high" Taylor numbers $(303 \leq \mathrm{Ta} \leq 1212)$ and relatively "high" axial Reynolds numbers $\left(\operatorname{Re}_{\mathrm{ax}} \leq 107\right)$, for relatively "big" CTS with a radial ratio $\eta=R_{\text {int }} / R_{\text {out }}=0.855$ and an aspect ratio $\Gamma=H / d=31.03$ (where $H$ is the CTS height and $d=\left(R_{\text {out }}-R_{\text {int }}\right)$ is the gap thickness), we realized a quantitative experimental study using standard and speed Velocimetry per Image of Particles (PIV) technique. The vortex structures for CTF with and without an "ascending" axial flow, according to the "direct protocol" i.e. The axial flow is superposed to an initial fully developed rotational flow were studied [5]. The vortex direction strongly depends on protocol history. The cartographies of velocity components are illustrated. The results mainly concern axial and radial velocity components. The cartographies of the vorticity $\omega$, and the detection criteria $Q$ and $\Gamma_{2}$ are presented and discussed. The alternating between positive and negative values of axial velocity component characterizes the presence of contrarotating vortices. This allows determining the axial wavelengths $(\lambda)$ for WTVF and MWTVF with and without axial flows. A same axial flow can have a stabilizing effect for a regime flow and a destabilizing effect for another. It enhanced the overlapping, the stretching, the folding or the breaking of vortices. From WTVF to MWTVF to TN, we illustrated that the vortices mixing is enhanced when the Taylor number increases due to vortices stretching and folding.

\section{NOMENCLATURE}

d Gap thickness of the Couette Taylor system (m).

F Kurtosis coefficient

$\mathrm{H} \quad$ Height of the Couette Taylor system (m).

$Q \quad$ Detection criteria

$\mathrm{R}_{\text {int }} \quad$ Inner Radius (m).

$\mathrm{R}_{\text {out }} \quad$ Outer Radius (m).

Re Reynolds number

$\mathrm{Re}_{\mathrm{ax}} \quad$ Definition2

$\operatorname{Re}_{\Omega} \quad$ Rotational Reynolds number

$S \quad$ Dissymmetry coefficient

Ta Taylor number

$\mathrm{u} \quad$ Axial component of the velocity $(\mathrm{m} / \mathrm{s})$.

$\mathrm{v} \quad$ Radial component of the velocity $(\mathrm{m} / \mathrm{s})$.

$\omega \quad$ Vorticity aspect ratio.

$\Gamma=\mathrm{H} / \mathrm{d}$ Aspect ratio.

$\Gamma_{2} \quad$ Detection criteria.

\section{INTRODUCTION}

Taylor-Couette CT flow is a rich fluid with series of clearly distinguishable flow regimes, depending on several operating 
parameters, from laminar Couette Flow CF via Wavy Taylor vortices flow WTVF [3], Modulated Wavy Taylor Vortex Flow MWTVF and chaotic flows to full turbulence. The superposition of an axial flow to Taylor-Couette flow is becoming frequently used in catalytic, photochemical, biochemical reactors, as well as in mass transfer processes such as extraction, tangential filtration, and crystallization [6,7]. Thus, the knowledge of the CT flows hydrodynamics without and with axial flows, known as Couette-TaylorPoiseuille flows CTPF, is necessary but it remains rather qualitative especially for high velocities. The majority of the studies reported in the literature deal with axial flows imposed to CTF for relatively low Taylor numbers and/or low axial flux rates [4]: $\operatorname{Re}_{\Omega}<50$ and $\operatorname{Re}_{\mathrm{ax}}<400$; [1] $: \mathrm{Re}_{\mathrm{ax}}<4$ ), or restricted to analytical or numerical studies [7-9]. Few experimental studies are found for vortices characterization of WTVF or MWTVF or Nascent Turbulence TN with moderated axial flows. However, it remains either qualitative studies using visualization for example or local studies using the ElectroDiffusion method to study the local mass transfer and wall shear rate evolutions and vortices impacts at the wall $[10,11]$. The studies of Couette-Taylor flows with axial flows carried with relatively small radial and aspect ratios [1-3]. For example, Moser et al., 2000 have visualized (2D slices) Taylor-Couette flows without and with superposed mean axial flux, using a snapshot FLASH spatial tagging sequence, with image acquisition times approximately half a second. Their visualization proved that spiral Poiseuille cells propagate at a higher velocity than the mean axial flow one. [5] used visualization via Kalliroscope particles and Electro-Diffusion (ED) Method to detect vortices and determine their impact at the wall i.e. mass transfer and wall shear stress evolutions.

In previous analytical works, an additional flow parameter is added $\gamma=\operatorname{Re}_{\mathrm{ax}} / \mathrm{Re}_{\Omega}$, where $\mathrm{Re}_{\mathrm{ax}}$ is the axial Reynolds number and $\operatorname{Re}_{\Omega}$ is the rotational Reynolds number. It has shown that the axial flow disrupts the structures and the transport properties of Taylor vortices by letting them travel in the direction of the axial flow if the axial flow is low. If the axial flow is important, the Taylor vortices break down into a spiral [12]. At this point, the symmetry of the Taylor-Couette flow is broken as the cells deform and overlap [3].

Almost of theses studies, no indication was found about the protocol i.e. if the rotational flow was imposed before or after the axial flow.

[8] proved, via an analytical approach then a numerical simulation taking into account the velocity-pressure coupling, that an axial flow and/or radial flow imposed to CTF alter the stability of the flow. At low $\mathrm{Re}_{\mathrm{ax}}$, the convective instability is axisymmetric, but convective helical modes with an increasing number of helices having a helicity opposite that of the base flow dominate as $\mathrm{Re}_{\mathrm{ax}}$ increases. [7] have proposed a model based on the assumption that the CTP flow is like a linked network of stirred tanks. Thus allows the study of mass exchange processes between Taylor vortices and the bypass stream. [3] used PIV measurements to demonstrate that the chaotic advection is the mechanism responsible for mixing in Wavy Vortex flow. In our previous experimental work using PIV [11], spatial distribution of the flow without and with a "low" axial flow were illustrated via the cartographies of vorticity $\omega$ as a swirling structures detection criterion, the criterion $Q$, and the instantaneous fields of $\Gamma_{2}$ criterion [13] obtained after the post-processing of the PIV data on a surface 7 layers. It shown that the axial flow superposed to WVF can have a stabilizing effect. Taylor vortices may move in axial flow direction. However, these flow characteristics strongly depend on protocols well described on our work. Although $Q$ criterion was developed for isolated vortex filaments, it is frequently applied to complex flows with interacting vortices. The objective of identifying the most intense structures usually justifies this application; however, the educed structure should be interpreted with care [15].

In this paper, we propose a quantitative study of CouetteTaylor-Poiseuille flows for relatively "high" Taylor numbers and axial Reynolds numbers, using PIV to characterize WTVF, MWTVF and TN flows with and without relatively high axial flows according to the "direct" protocol. The coherent structures are detected using the detection criteria $Q$ and $\Gamma_{2}$.

\section{EXPERIMENTAL APPARATUS AND PIV TECHNIQUE}

The experimental installation of Couette-Taylor-Poiseuille is constituted of two coaxial cylinders, a rotating inner one (the rotor) and a fixed outer one (the stator) (Figure 1). The inner cylinder has a radius $R_{1}=85.5 \mathrm{~mm} \pm 0.2$. The outer cylinder has a radius $R_{2}=100 \mathrm{~mm} \pm 0.2$. The height of the rotating system is $\mathrm{H}=450 \mathrm{~mm} \pm 0.5$. The corresponding radius ratio is $\eta=0.855$ and the aspect ratio is $\Gamma=31$. The system contains also a storage tank, a pump; a by-pass; a flowrate control valve, a flowmeter; a CT feeder tank; a drain valve; and an electrical motor which assure the inner cylinder motion. The Velocimetry per Image of Particles (PIV) laser technique was used to determine the instantaneous velocity field.

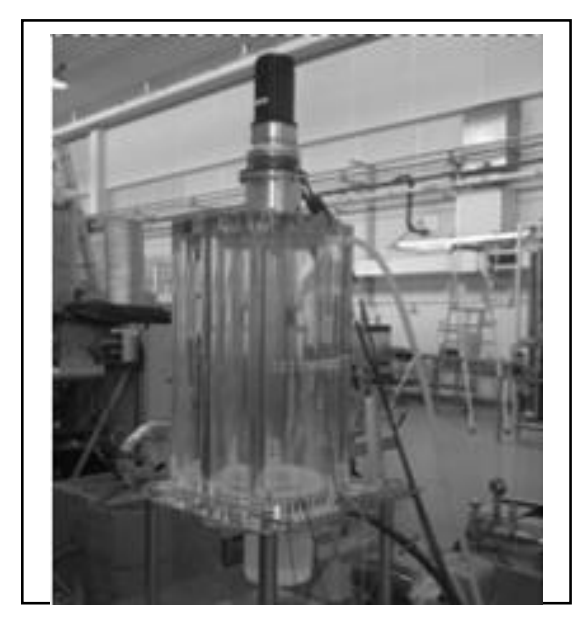


FIGURE 1. THE EXPERIMENTAL INSTALLATION

\section{RESULTS AND DISCUSSION}

\section{Effect of axial flow on velocity}

\subsection{Wavy Vortex Flow without and with an axial flow.}

The cartography of the iso-lines values of the axial and radial velocities at a Taylor number equals to $\mathrm{Ta}=303$, without and with an axial upflow corresponding to $\mathrm{Re}_{\mathrm{ax}}=73.17$ are shown in Figures 2 and 3. At $\mathrm{Re}_{\mathrm{ax}}=0$, there are positive and negative values of velocity axial component that characterize the presence of well developed contra-rotating vortices. For a height of $19.5 \mathrm{~d}$, the cartographies obtained by PIV standard $(15 \mathrm{~Hz})$ show the existence of 7.5 pairs of contra-rotating vortices well developed.
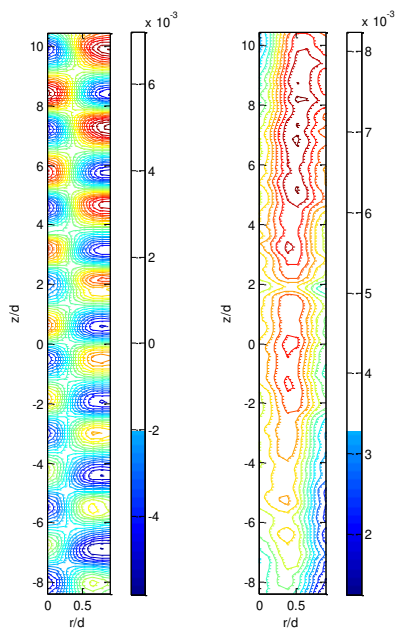

FIGURE 2. ISO-LINES VALUES OF THE AXIAL VELOCITY COMPONENT FOR Ta=303: A) $\operatorname{Re}_{a x}=0$; B) $\operatorname{Re}_{a x}=73.17$
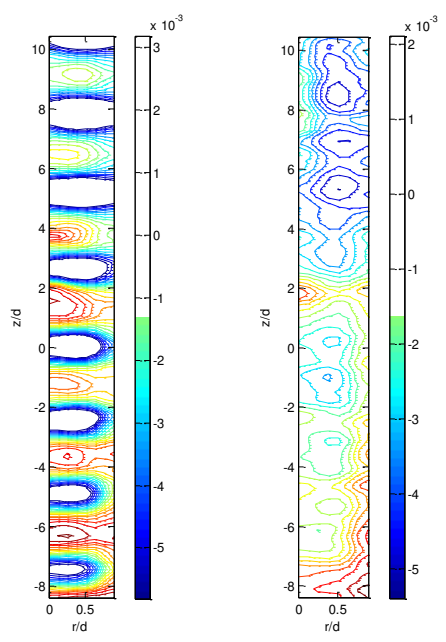

FIGURE 3. ISO-LINES VALUES OF THE RADIAL VELOCITY COMPONENT FOR Ta=303: A) $\mathrm{Re}_{\mathrm{ax}}=0$; B) $\mathrm{Re}_{\mathrm{ax}}=73.17$

At for $\mathrm{Re}_{\mathrm{ax}}=73.17$, the axial flow causes the overlapping, the stretching then the breaking of vortices (Figure 2). The WV flow is destabilized under the effect of a relatively high axial flow. While, for an axial flow 23 times smaller $\left(\mathrm{Re}_{\mathrm{ax}}=3.15\right)$, the axial flow has a stabilizing effect even if it leads to a slight inclination of the axial velocity component and an increase of the wavelength of the axial vortices which follow the axial flow direction [11].

\subsection{Modulated Wavy Vortex Flow without and with an axial flow.}

The figures 4 and 5 present the iso-values lines of the radial and axial velocity components for $\mathrm{Ta}=606$ without and with an axial flow corresponding to an axial Reynolds number of $\operatorname{Re}_{\mathrm{ax}}=101$. The wavelength Taylor vortices increases to $\lambda=$ 3.1d, for $\mathrm{Ta}=606$ and $\mathrm{Re}_{\mathrm{ax}}=0$. At a fully superposed axial flow corresponding to $\mathrm{Re}_{\mathrm{ax}}=101$ superposed to the initial flow $(\mathrm{Ta}=606)$, there are a waviness then an overlapping of the vortices which have the same direction as the axial flow. The axial flow, characterized by a Reynolds number $\mathrm{Re}_{\mathrm{ax}}=101$, causes the destabilization and the breaking of modulated Taylor vortices.
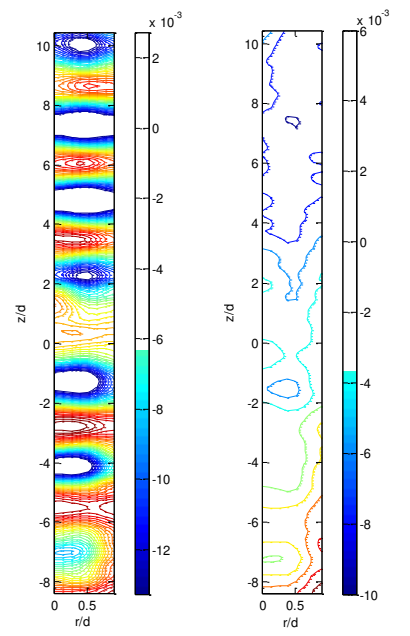

FIGURE 4. ISO-LINES VALUES OF THE RADIAL VELOCITY COMPONENT FOR Ta=606: A) $\mathrm{Re}_{\mathrm{ax}}=0$; B) $\mathrm{Re}_{\mathrm{ax}}=101$ 


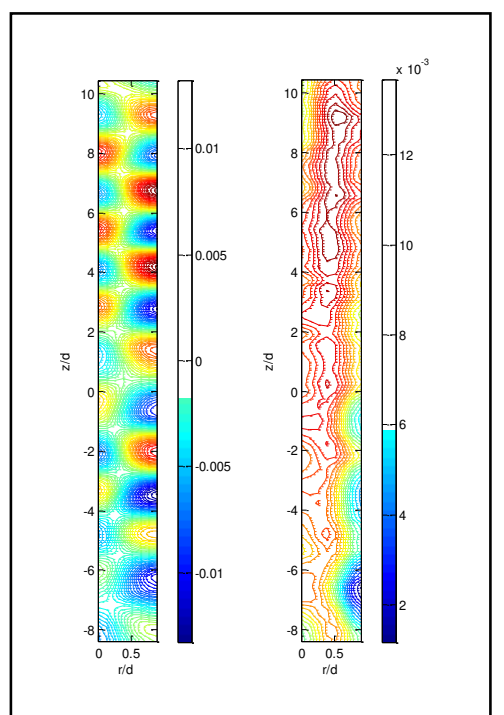

FIGURE 5. ISO-LINES VALUES OF THE AXIAL VELOCITY COMPONENT FOR Ta=606: A) $\operatorname{Re}_{a x}=0$; B) $\operatorname{Re}_{a x}=101$

\subsection{Modulated Wavy Vortex Flow without and with an axial flow}

\subsubsection{Effect of high axial flow on Velocity components}

The iso-lines values of the radial and the axial velocity components for $\mathrm{Ta}=1212$, without and with an axial flow corresponding to an axial Reynolds number $\mathrm{Re}_{\mathrm{ax}}=107$, are presented respectively in Figures 6 and 7. Comparing Turbulent Nascent TN flow cartographies without an axial flow to those with a relatively high axial flow $\left(\operatorname{Re}_{a x}=107\right)$, we proved that the Taylor vortices persist despite the relatively high axial flow. However, the vortices are stretched and folded. They emphasize the presence of the jet on the inlet region (inflow) and the outlet region (outflow). The jets generate a thinning of the boundary layer on the inflow and the outflow areas. This is manifested by an inclination and stretching of Taylor vortices due to shear. This inclination is accentuated when the Taylor number increases i.e. for the nascent turbulence (TN). While comparing the axial velocity cartographies presented for different flow regimes, we found that when the Taylor number increases, there is an increase of the extrema of the average axial velocity, and a shift of these extrema is more accentuated towards the outer cylinder wall.

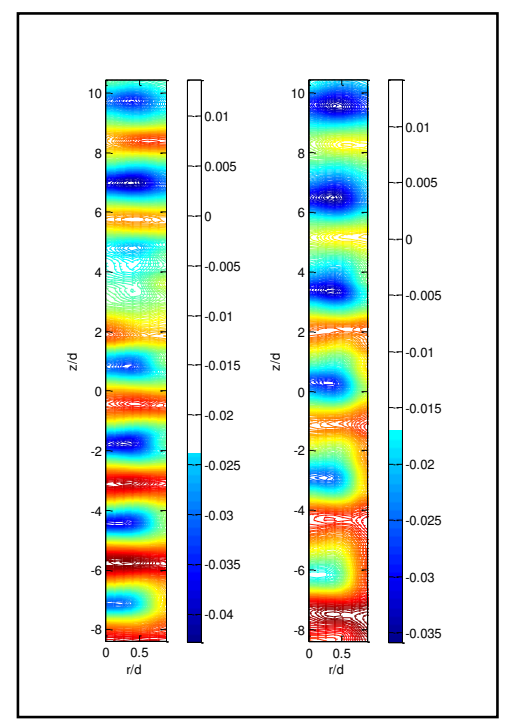

FIGURE 6. ISO-LINES VALUES OF THE RADIAL VELOCITY COMPONENT FOR Ta=1212: A) $\operatorname{Re}_{\mathrm{ax}}=0$; B) $\operatorname{Re}_{\mathrm{ax}}=101$

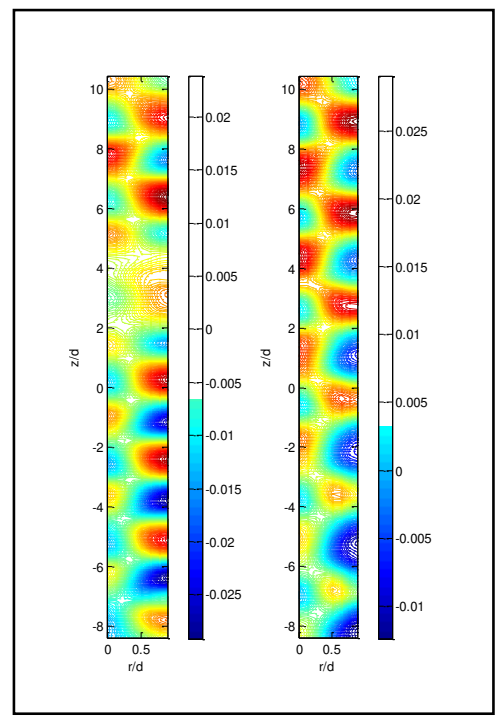

FIGURE 7. ISO-LINES VALUES OF THE AXIAL VELOCITY COMPONENT FOR Ta=1212: A) $R_{a x}=0$; B) $R_{a x}=107$

\section{Effect of axial flow on coherent structure criteria : vorticity, $Q$ and criteria $\Gamma_{2}$}

\subsection{Wavy Vortex Flows with and without axial flow.}

The vorticity $\omega$ cartographies for $\mathrm{Ta}=303$ and respectively for $\mathrm{Re}_{\mathrm{ax}}=0$, and $\mathrm{Re}_{\mathrm{ax}}=73.17$ are presented in Figure 8. While, the spatial distributions of the flow obtained for the criterion $Q$ are shown in Figure 9. This allows us to deduce the vortex characteristics such as numbers, forms, wavelengths, intensities, etc. For a height of $\mathrm{z} / \mathrm{d}=19.5$, we visualized by PIV 7.5 pairs of well developed contra-rotating vortices. The $Q$ criterion cartographies are used to determine the vortex wavelength. The 
latter is equals to $\lambda \approx 2.6 \mathrm{~d}$ for $\mathrm{Ta}=303$ and $\mathrm{Re}_{\mathrm{ax}}=0$. For $\operatorname{Re}_{\mathrm{ax}}=73.17, Q$ criterion cartographies illustrate the presence of non-developed vortices of smaller size. The axial flow generate an overlapping of the initial vortices of $2.6 \mathrm{~d}$ wavelength to smaller one. This is not detectible using the vorticity. The use of the vorticity $\omega$ as criterion for vortices detection is not suitable in the presence of significant shear wall. In this case, the $\Gamma_{2}$ criterion is usually preferable, because it is not sensitive to shear. Details about the determination of the instantaneous wall shear stress are available on [5].

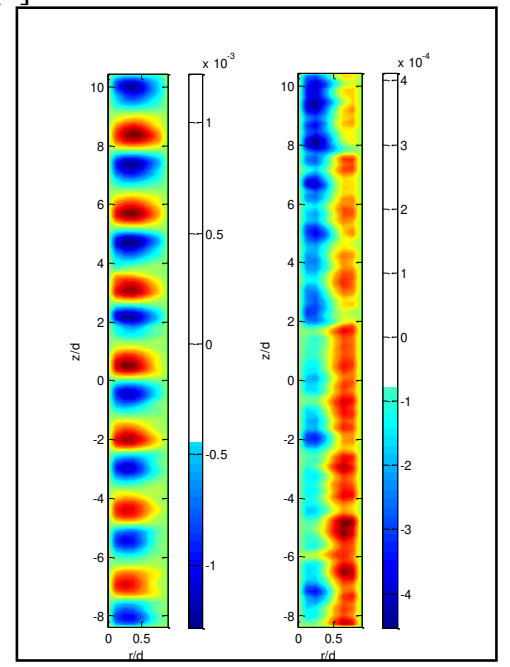

FIGURE 8. THE VORTICITY $\omega$ CARTHOGRAPHIES FOR $\mathrm{Ta}=303$ : A) $\mathrm{Re}_{\mathrm{ax}}=0$; B) $\mathrm{Re}_{\mathrm{ax}}=73.17$

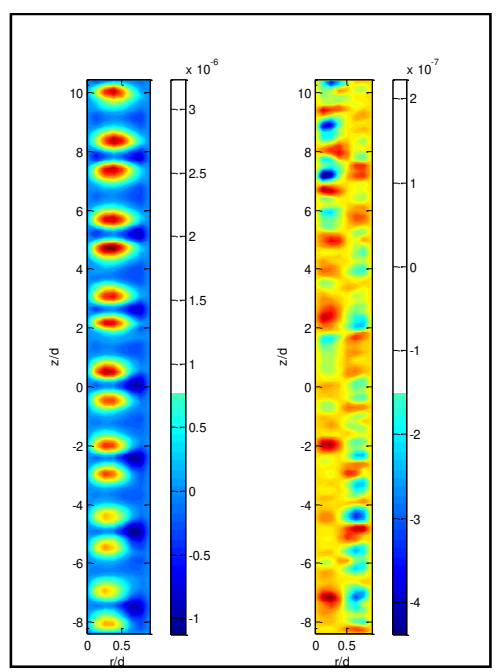

FIGURE 9. THE $Q$ CRITERIA CARTHOGRAPHIES FOR $\mathrm{Ta}=303: \mathrm{A}) \mathrm{Re}_{\mathrm{ax}}=0$; B) $\mathrm{Re}_{\mathrm{ax}}=73.17$

The instantaneous fields of the $\Gamma_{2}$ criterion obtained after the post-treatment of PIV data on a 7 layer area and for $\mathrm{Ta}=303$ and respectively for $\mathrm{Re}_{\mathrm{ax}}=0$, and $\mathrm{Re}_{\mathrm{ax}}=73.17$ are illustrated in Figure 10. Indeed, these figures show the spatial distribution of Taylor vortices along the gap. When the axial flow becomes large, the vortices become increasingly overlapped and unstructured.

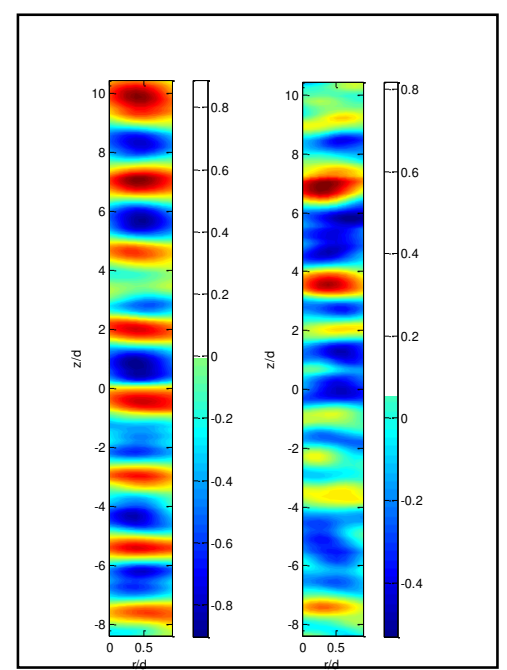

FIGURE 10. THE $\Gamma_{2}$ CRITERIA CARTHOGRAPHIES FOR $\mathrm{Ta}=303: \mathrm{A}) \mathrm{Re}_{\mathrm{ax}}=0$; B) $\operatorname{Re}_{\mathrm{ax}}=73.17$

\subsection{Modulated Wavy Vortex Flows with and without axial flow.}

The fields of vorticity and the $Q$ criterion are shown respectively in Figure 11 and 12 for a flow without an axial flow for a MWVF with an axial flow corresponding to a Reynolds number $\mathrm{Re}_{\mathrm{ax}}=101$.

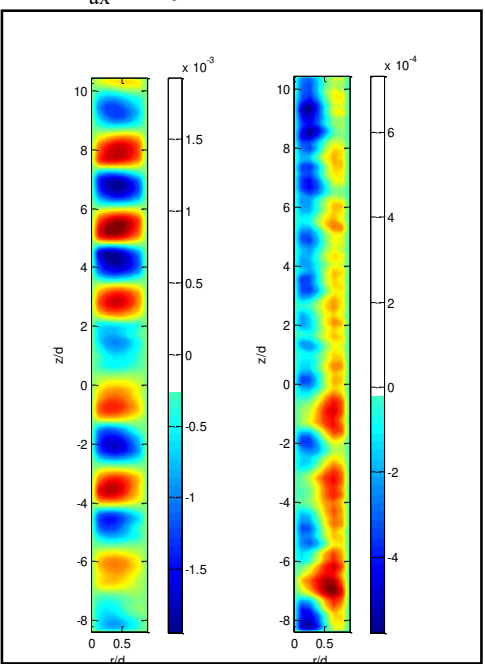

FIGURE 11. THE VORTICITY $\omega$ CARTHOGRAPHIES FOR $\mathrm{Ta}=606$ : A) $\mathrm{Re}_{\mathrm{ax}}=0$; B) $\mathrm{Re}_{\mathrm{ax}}=101$

While, the field of the instantaneous $\Gamma_{2}$ criterion obtained after the PIV data post- processing on a 7 surface layer is provided in Figure 13. The different mappings characterize the counterrotating vortices present at a height of $19.5 \mathrm{~d}$. They highlight the characteristics of vortex in shape, centre and wavelength. The wavelength of Taylor vortices is $\lambda \approx 3.1 \mathrm{~d}$ for $\mathrm{Ta}=1212$ and $\mathrm{Re}_{\mathrm{ax}}=0$. Vortices are identified by positive iso- values 
(Figure 11 and 12). Their centres are identified by the maximum values of the $Q$ criterion. For $\operatorname{Re}_{\mathrm{ax}}=101$, the $Q$ criterion and the instantaneous $\Gamma_{2}$ detect the overlapping of the modulated Taylor vortices.

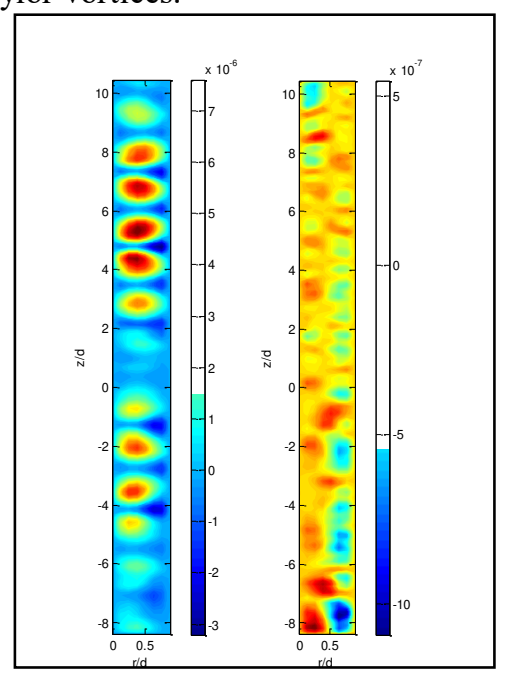

FIGURE 12. THE $Q$ CRITERIA CARTHOGRAPHIES FOR $\mathrm{Ta}=606$ : A) $\mathrm{Re}_{\mathrm{ax}}=0$; B) $\mathrm{Re}_{\mathrm{ax}}=101$

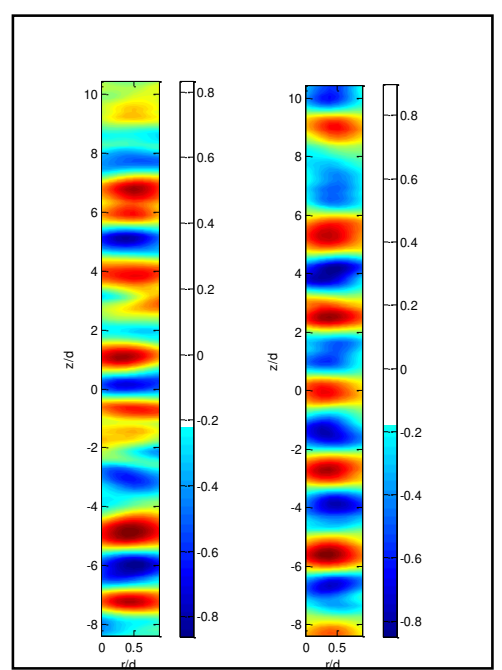

FIGURE 13. THE $\Gamma_{2}$ CRITERIA CARTHOGRAPHIES FOR $\mathrm{Ta}=606: \mathrm{A}) \mathrm{Re}_{\mathrm{ax}}=0$; B) $\mathrm{Re}_{\mathrm{ax}}=101$

\subsection{Nascent turbulent flows with and without axial flow.}

At the instability threshold characterizing the transition between MWVF and nascent turbulent flow, the vorticity field, the $Q$ criterion and the $\Gamma_{2}$ criterion obtained after PIV data postprocessing on 7 layers are presented, respectively for $\mathrm{Re}_{\mathrm{ax}}=0$ and $\mathrm{Re}_{\mathrm{ax}}=107$ (Figure 14 to Figure 16). The cells appear in the region where the flow is dominated by the rotational tensor. Vortices are easily identifiable by the positive $Q$ iso-values. While their centres are identified by the maximum values of this criterion. The vortex wavelength is $\lambda=3.25 \mathrm{~d}$.

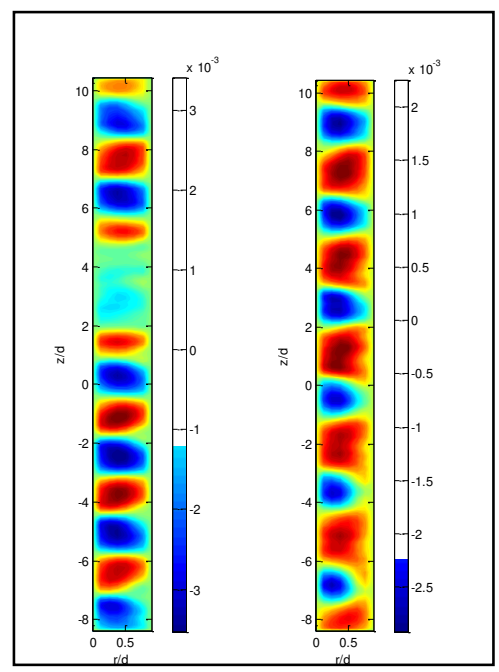

FIGURE 14. THE VORTICITY $\omega$ CARTHOGRAPHIES FOR $\mathrm{Ta}=1212$ : A) $\operatorname{Re}_{\mathrm{ax}}=0$; B) $\mathrm{Re}_{\mathrm{ax}}=107$

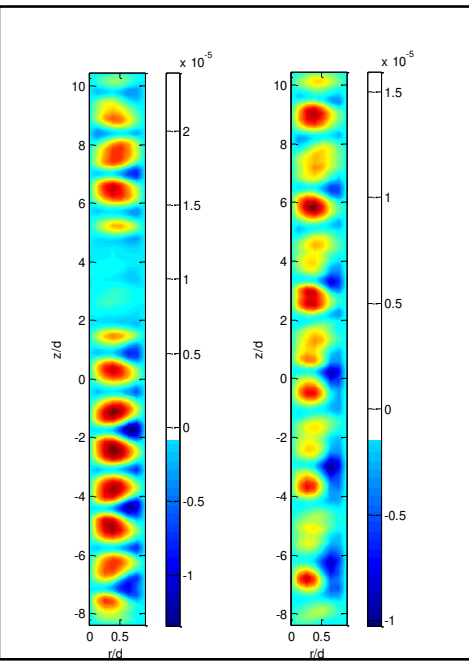

FIGURE 15. THE $Q$ CRITERIA CARTHOGRAPHIES FOR $\mathrm{Ta}=1212: \mathrm{A}) \mathrm{Re}_{\mathrm{ax}}=0$; B) $\mathrm{Re}_{\mathrm{ax}}=107$

The different criteria cartographies illustrate that a relatively high axial flow is insufficient to break nascent turbulence vortex. However, comparing the TN wavelength vortex to the MWVF one without axial flow, it was found that the vortices become more stretched. Their wavelength increases from $\lambda=3.1 \mathrm{~d}(\operatorname{Reax}=0)$ to $\lambda=3.25 \mathrm{~d}(\operatorname{Reax}=107)$. A high axial flow can have a stabilizing effect on TN.

Comparing the TN flows with a relatively important axial flow (Reax $\approx 100$ ) to MWTVF, we proved that the same axial flow is sufficient to break the MWT vortex, but insufficient to destabilize the nascent turbulent regime where vortices persist along the gap. However, the vortices are more scattered because of the axial flow. 


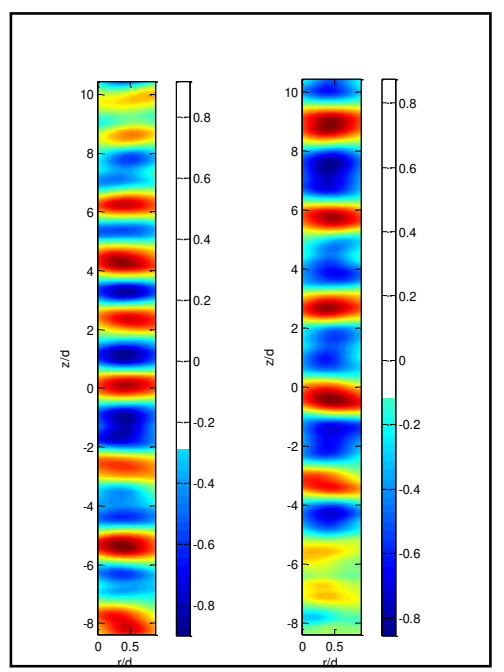

FIGURE 16. THE $\Gamma_{2}$ CRITERIA CARTHOGRAPHIES FOR $\mathrm{Ta}=1212: \mathrm{A}) \mathrm{Re}_{\mathrm{ax}}=0$; B) $\mathrm{Re}_{\mathrm{ax}}=107$

\section{CONCLUSION}

Relatively high axial flows were superposed to a fully developed Wavy Vortex flows, Modulated Wavy Vortex Flows and Nascent flows (the direct protocol). The vortices direction strongly depends on the axial flow direction for all the cases studied. Thus, the vortices follow the axial up flow, which have a stabilizing effect on vortices or a destabilizing effect according to the torsional flow (Ta) and axial flow (Re) ratio. In such destabilizing cases, the vortices are either folded or stretched or overlapped or completely destructed. The vortices wavelengths, their sizes, centres, intensities are determined using the coherent detection criteria: the vorticity field, the $Q$ criterion and the $\Gamma_{2}$ criterion obtained after PIV data postprocessing on 7 layers.

\section{ACKNOWLEDGMENTS}

This work was supported by the laboratory GEPEA (University of Nantes) and the "Agence Nationale de la Recherche (ANR), France", by the grant $\mathrm{n}^{\circ}$ ANR-08-BLAN-0184-02 and the European Commission within the International Research Staff Exchange Scheme (IRSES) in the 7th Framework Programme FP7/2014-2017/ under REA grant agreement $n^{\circ} 612230$. These supports are gratefully acknowledged.

\section{REFERENCES}

[1] Dumont, E., Fayolle, F., Sobolik, V., Legrand, J., 2002. "Wall shear rate in the Taylor-Couette-Poiseuille flow at low axial Reynolds number". Int. Journal of Heat and Mass Transfer, 45(3), pp. 679-689.

[2] Moser, K. W., Raguin, L. G, Harris, A., Douglas, Morris H., Georgiadis, J., Shannon, M., Philpott, M., 2000. "Visualization of Taylor-Couette and spiral Poiseuille flows using a snapshot FLASH spatial tagging sequence”. Magnetic Resonance Imaging 18, pp. 199-207.

[3] Akonur, A., Lueptow, R. M., 2002. "Chaotic mixing and transport in wavy Taylor-Couette flow". Physica D 167, pp. 183-196.

[4] Leclercq, C., Pier, B., Scott, J., 2013. "Absolute instabilities in eccentric Taylor-Couette-Poiseuille flow". 14th European turbulence conference, Lyon, France.

[5] Berrich, E., Aloui, F., Legrand, J., 2012. "On the Stability of Taylor - Couette Flow With Axial Flow". ASME 2013, Paper No. FEDSM2012-72235, pp. 899-908; doi:10.1115/FEDSM2012-7223.

[6] Resende, M. M., Vieira, P. G., Sousa, Jr R., Giordano, R. L. C., Giordano, R. C., , 2004. "Estimation of mass transfer parameters in a Taylor-Couette-Poiseuille heterogeneous reactor". Brazilian Journal of Chemical Engineering, 21 (02) pp. $175-184$.

[7] Syed, A., Fruh, W-G., 2003. "Modelling of mixing in a Taylor-Couette reactor with axial flow". Journal of Chemical Technology and Biotechnology, 78 (2-3), pp. 227-235. $10.1002 /$ jctb. 758 .

[8] Martinand, D., Serre, E., and Lueptow, R.M., 2009. "Absolute and convective instability of cylindrical Couette flow with axial and radial flows". Physics of fluids 21, 104102.

[9] Leclercq, C., Pier, B., Scott J., 2013. "Temporal stability of eccentric Taylor Couette Poiseuille fow”. J. Fluid Mech. 733, pp. 68-99. Cambridge University Press 2013 68, doi:10.1017/jfm.2013.437.

[10] Kristiawan, M., Tomas, Jirout T., El Faye, A., Sobolik, V., 2011. "Wall shear rate components for wavy Taylor-Couette flow". Experimental Thermal and Fluid Science 35, pp. 13041312.

[11] Berrich, E., Aloui, F., Legrand, J., 2012. "Synchronization between PIV and Electro-diffusion techniques for the characterization of Wavy Taylor Vortex flows with axial flows". Turbulence, Heat and Mass Transfer 7, 2012, DOI: 10.1615/ICHMT. ProcSevIntSymp TurbHeatTransf Pal.590.

[12] Takeuchi, D.I., Jankowski, D.F., 1981. "A numerical and experimental investigation of the stability of spiral Poiseuille flow". Journal of Fluid Mechanics, 102, pp. 101-126

[13] Graftieaux, L., Michard, M., Grosjean, N., 2001. "Combining PIV, POD and vortex identification algorithms for the study of unsteady turbulent swirling flows". Measurement Science and Technology 12, pp. 1422-1432.

[14] Chakraborty, P., Balachandar, S. and Adrian, R. J., 2005. "On the relationships between local vortex identification schemes". Journal of Fluid Mechanics, 535, pp. 189-214.

[15] Batchelor, GK., 1953. "The Theory of Homogeneous Turbulence". Cambridge, UK: Cambridge, Univ. Press. 